\title{
SIMULATION OF OPTICAL PROPERTIES OF SI WIRE CELLS
}

\author{
Pietro P. Altermatt, ${ }^{1,2}$ Yang Yang, ${ }^{2,3}$ Thomas Langer, ${ }^{4}$ Andreas Schenk, ${ }^{5}$ Rolf Brendel ${ }^{1,2}$ \\ ${ }^{1}$ Dep. Solar Energy, Inst. Solid-State Physics, Leibniz University of Hannover, Appelstr. 2, 30167 Hannover, Germany \\ 2 Institute for Solar Energy Research Hamelin (ISFH), Am Ohrberg 1, 31860 Emmerthal, Germany \\ 3 Institute for Solar Energy Systems, Sun Yat-Sen University, 510006, Guangzhou, China \\ ${ }^{4}$ Dep. Surface Science, Inst. Solid-State Physics, Leibniz University of Hannover, Appelstr. 2, 30167 Hannover, Germany \\ ${ }^{5}$ Integrated Systems Laboratory, ETH Zurich, Gloriastr. 35, 8092 Zurich, Switzerland
}

\begin{abstract}
We solve the Maxwell equations to quantify the amount of photo-generation in Si solar cells consisting of arrays of wires instead of bulk thin-films. Published transmission and reflectance measurements suggest that an array of $\mathrm{Si}$ wires absorbs sunlight very effectively due to strong diffraction and scattering. However, a detailed theoretical understanding and quantification of the actual photogeneration is only in its initial stage. In our simulations, the geometrical parameters of the wires are synthesized by means of cluster simulations. Applying the finite element method, we are able to compute randomly aligned wires within manageable time limits and affordable computer capacity. We show that Si wires have strong photonic properties. For example, our simulations surpass the Lambertian limit (for isotropically incident light) at $\lambda=1000$ $\mathrm{nm}$, as has been reported in many experiments.
\end{abstract}

\section{MOTIVATION}

Recently, Si solar cells consisting of arrays of wires instead of bulk thin-films have been fabricated. Transmission and reflectance measurements suggest that an array of Si wires absorbs sunlight very effectively due to strong diffraction and scattering [1-5]. The wires are thinner than the wavelength of sunlight and rather closely packed together, so the optical properties of wire arrays are largely determined by the diffuse multiple scattering of light. This is the reason why the optical properties cannot be successfully calculated by means of effective medium theories [2]. On top of this, the high index of refraction of Si makes wire arrays strongly photonic, even if the wires are only a few $\mathrm{nm}$ thin [2]. This may be one of the reasons why the measured transmittance is usually low even for photons with energies below the band gap, where bandto-band absorption is absent.

To optimize the structure of wire cells, it may be insufficient to minimize reflectance and transmittance: there is a large amount of scattering present and, depending on the cell structure, there may be considerable amounts of parasitic absorption, e.g. due to photonic properties. Consequently, one has to focus on maximizing the photo-generation rate $\mathrm{G}$.
While data on the transmittance and reflectance of wire cells can be obtained relatively easily, the quantification of $G$ in the wire cells is difficult. For example, the extraction of $G$ from quantum efficiency measurements would require knowledge not only of the optical properties, but also of the carrier collection efficiency.

It is therefore the goal of this work to aid in the theoretical understanding and quantification of the photogeneration rate in Si wire arrays by means of solving the Maxwell equations in three dimensions.

\section{THE GEOMETRICAL MODEL}

The arrays are often fabricated with the help of selforganisational effects. For example, a metal film is tempered and disintegrates into many clusters, which are used as catalysts for growing the wires. Consequently, the wires are positioned with some degree of randomness. Due to the photonic properties of wire arrays, the positions of the wires influence the simulations considerably and need be carefully chosen.

If a uniform probability distribution is selected to calculate the positions of the wires, the rather regular patterns that have been identified in experiments cannot be reproduced, as is shown in Figure 1. This is because, in reality, the randomness is superimposed on a nonrandom physical law, namely the minimization of the surface potential during clustering. We therefore extract the positions of the wires from a clustering simulation. We do not intend to simulate the course of the clustering; rather, we are interested in a realistic arrangement of the wires once the surface potential is near minimum. Therefore, we use the Metropolis-Hastings algorithm [6] with a simple Lennard-Jones-(4,2)-Potential,

$$
V(r)=4 \varepsilon\left[\left(\frac{\sigma}{r}\right)^{4}-\left(\frac{\sigma}{r}\right)^{2}\right]
$$

with $\varepsilon=40-75$ and $\sigma=300-400 \mathrm{~nm}$. The initial state is prepared by simply positioning the wires randomly. During each simulation step, the coordinates of the wires are changed to a random position within a region of $100 \mathrm{~nm}$ around their previous positions. The Hamiltonian is calculated after each step by considering only the potential energy of the system, given by the placement of the wires. 
A new position of a wire is rejected if the potential has risen by too much. It turned out that about $30 \%$ of cases yielded a higher potential, as to prevent the system from being too homogeneous. An arrangement close to minimal potential is shown in Figure 2.
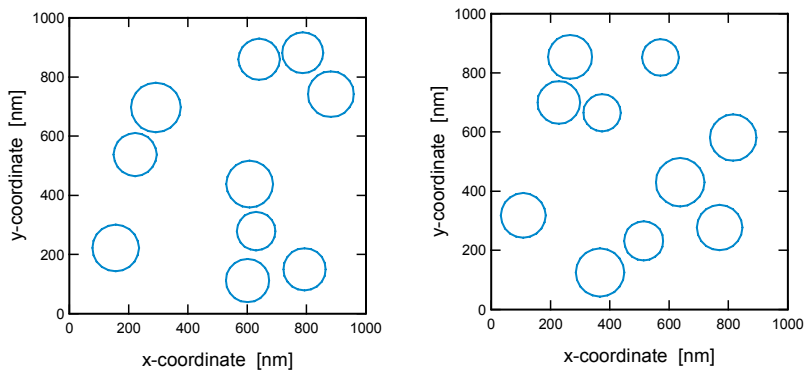

Figure 1. If the positions of the wires are chosen with a uniform random distribution, the experimentally realised, rather regular patterns cannot be reproduced. Ten wires are shown in a simulation domain of $1 \times 1 \mu \mathrm{m}^{2}$.

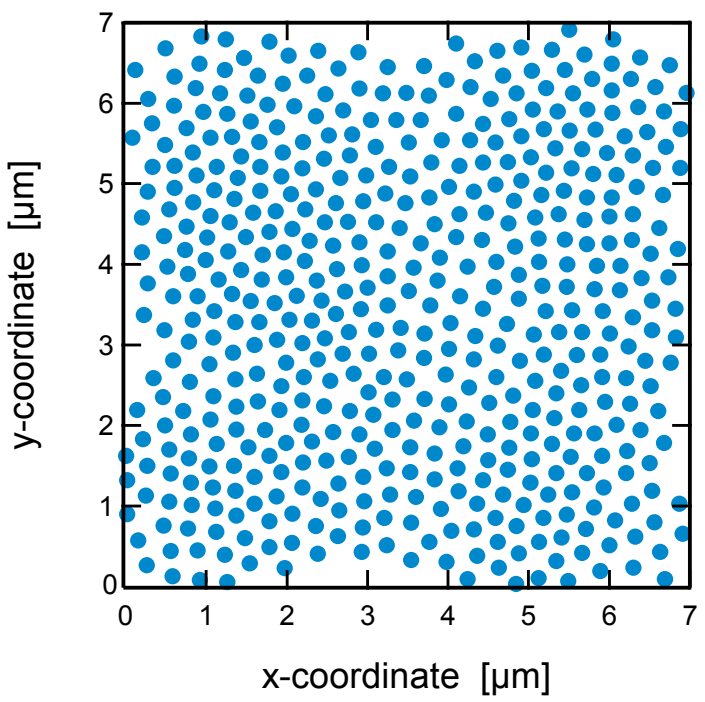

Figure 2. The positions of the wires calculated with a clustering simulation, in a state where the potential is near minimum. The domain here is 7 times larger than in Figure 1 , i.e. there are 490 wires in an area of $7 \times 7 \mu \mathrm{m}^{2}$.
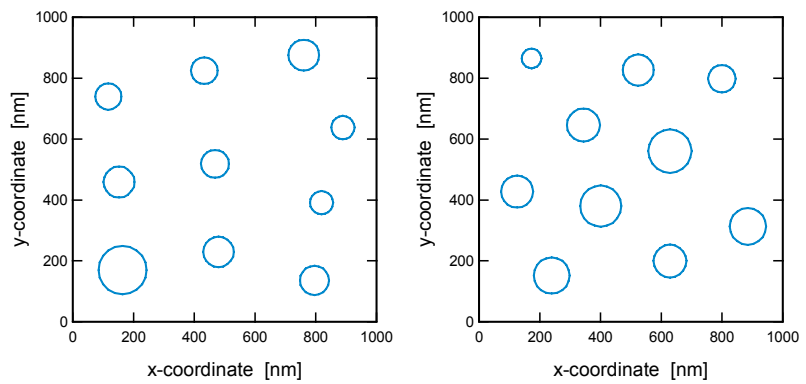

Figure 3. Two domains with $1 \times 1 \mu \mathrm{m}^{2}$ area, chosen from Figure 2, with each containing ten wires.
From this clustering simulation, various domains with $1 \times 1$ $\mu \mathrm{m}^{2}$ area are chosen as input for the optical simulation; see Figure 3 for two examples. When choosing these areas, one has to bear in mind that the optical simulations have periodic boundary conditions, so they imitate an infinite array of identical "tiles" with $1 \times 1 \mu \mathrm{m}^{2}$ area. We choose the domains from locations where ten wires, instead of nine, fit in the area because domains with nine wires have a too regular structure as to represent the experiment effectively.

Once the positions of the wires are chosen, the wires' diameters, lengths, and tilt-angles are chosen with a normal probability distribution, while a uniform probability distribution is used to calculate the azimuth angles of the tilt. In effect, we use pseudo-random numbers, because we discard random sets with penetrating wires or with wires crossing the domain boundary.

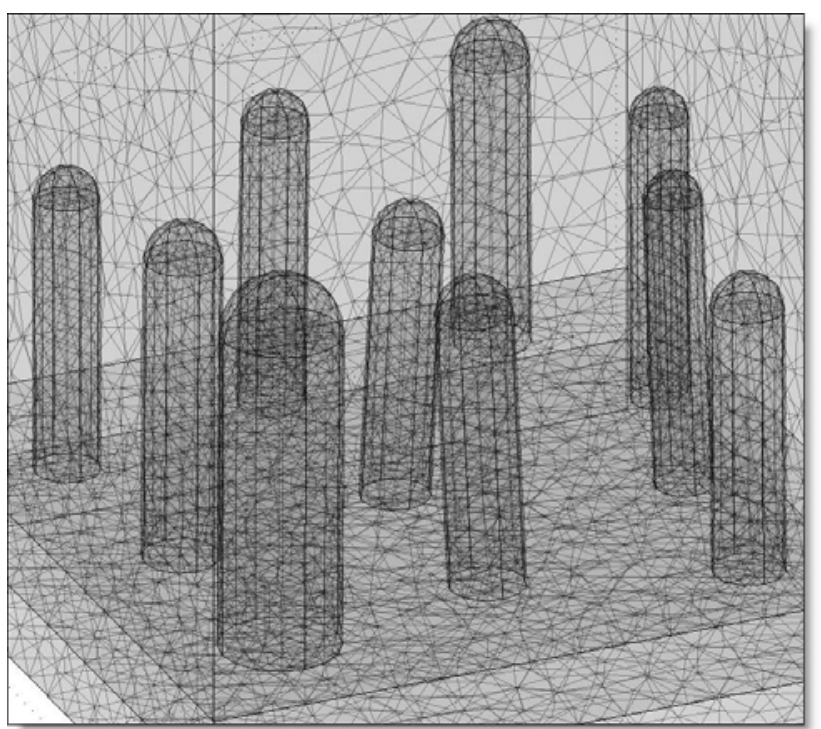

Figure 4. An example of the geometry and the tetrahedral mesh, generated with the finite-element modeling software Comsol [7].

The meshing and the simulations are done with Comsol [7], a versatile finite-element modeling software. The maximum size of the tetrahedral mesh elements must not exceed 1/10th of the wavelength of excitation in each optical medium. Otherwise, the solution of the Maxwell equations has insufficient numeric precision. Our shortest wavelength (in a vacuum) is $600 \mathrm{~nm}$, so we mesh the surroundings of the wires with maximally $60 \mathrm{~nm}$ mesh size, and the wires themselves with maximally $15 \mathrm{~nm}$ mesh size (because silicon has a refractive index of nearly 3.9 at a vacuum wavelength of $600 \mathrm{~nm}$ ). With these settings, the total number of mesh points in our domain is kept below 400 000, and results in 2.5 million degrees of freedom for solving the Maxwell equations. Hence, our computer system with $128 \mathrm{~GB}$ of RAM is sufficient for this task. At longer wavelengths, the mesh size is adapted to the optical parameters, resulting in fewer mesh points, which saves computing time. 


\section{THE OPTICAL MODEL}

Because the geometric features are smaller than the wavelength of sunlight, geometric ray tracing is not feasible. Also, it has been demonstrated [2] that the optical properties of wire arrays cannot be satisfactorily described using effective-medium theories. Instead, photonic properties and scattering must be taken into account. We therefore solve the fully coupled set of Maxwell equations, coupled with the materials equations, in three dimensions, using the Finite-Element (FE) approach. Because both the monochromatic excitation and the optical response (reflection, diffraction, scattering) are time-harmonic with frequency $\omega$, the fields can be factorized in a spacedependent (amplitude) part and a time-dependent (frequency) part:

$$
\vec{E}(\vec{r}, t)=\vec{E}(\vec{r}) e^{i \omega t} \quad \vec{H}(\vec{r}, t)=\vec{H}(\vec{r}) e^{i \omega t} .
$$

Inserting this into the Maxwell equations yields the Helmholtz equations:

$$
\begin{aligned}
& \nabla \times\left(\mu^{-1} \nabla \times \vec{E}\right)-\omega^{2} \varepsilon_{c} \vec{E}+i \omega \sigma \vec{E}=0 \\
& \nabla \times\left(\varepsilon_{c}^{-1} \nabla \times \vec{H}\right)-\omega^{2} \mu \vec{E}+i \omega \sigma \vec{H}=0 .
\end{aligned}
$$

Hence, we solve the equations in the time-harmonic approach, i.e. only for the amplitudes of $E(\vec{r})$ and $H(\vec{r})$. This approach differs from finite-difference time-domain (FDTD) simulations [8], which are a common benchmark in optical simulations. We have demonstrated in [9] that these two approaches deliver identical results within numerical precision.

We like to stress that selecting appropriate boundary conditions (BCs) is an issue, because the geometry of the wires is random. For example, the usual periodic boundary conditions cause artificial diffraction patterns, although the wires are positioned randomly as described above. It is common to choose absorbing BCs for strongly diffracting geometries and to solve only for the scattered field. However, with this approach, we would simulate only an isolated $\mu \mathrm{m}^{2}$ of wire array instead of an infinite array, and would introduce intolerable errors.

We found that artificial effects are reduced to a tolerable limit if (i) Floquet conditions are applied to the boundaries at the sides of the simulation domain, and (ii) wires too close to the sides of the Floquet $\mathrm{BC}$ are discarded. Periodic Floquet BCs ensure that the incoming sunlight with wave vector $\vec{k}$ - coming from a tilted angle is appropriately phase-shifted when switching from the outgoing side (at point 1) to the incoming side (at point 2):

$$
E(2)=E(1) e^{-i \vec{k}^{*} \vec{d}}
$$

where $\vec{d}$ is the translational vector from point 1 to 2 . This ensures a correct pattern of the incoming radiation in the absence of the wires. However when wires are present, the incoming sunlight gets strongly scattered, so the resulting wave vector $\vec{k}$ depends on the solution and cannot be anticipated for inserting it into the BC. Nor is it homogeneous within the simulation domain. Hence, any periodic boundary condition is not optimum for strong scattering effects. Because the wire arrangements are random, the "wrong" phase shift of scattered light, caused by the Floquet BCs, influences the simulation results randomly as well and will cancel out once a high enough number of random wire arrangements are simulated and the extracted results are averaged. In this way, some compromises are made to the geometrical setup while the errors due to BCs of both incoming and scattered radiation are strongly reduced.

The incoming sunlight is generated at the top boundary as planar wave with a single frequency. The bottom boundary is fully absorbing to mimic transmission further into the glass substrate.

The optical generation within $\mathrm{Si}$ is extracted from the time-averaged resistive heating, i.e. it is assumed that all absorption is caused by band-to-band absorption. The resistive heating can be converted to the number of photogenerated electron-hole pairs, because sunlight with only one single frequency is simulated at a time.

The reflectance and transmittance values are extracted by comparing each simulation with a simulation with the same mesh but in vacuum. In this way, the incoming time-averaged energy flow $l_{i}$ at the top boundary can be extracted as well as the reflected time-averaged energy flow $I_{r}$ through the top boundary, and the reflectance

$$
R=\frac{I_{r}}{I_{i}}
$$

can be calculated. Similarly, the transmittance can be calculated from the outgoing time-averaged energy flow $I_{t}$ at the bottom boundary compared to the simulation in vacuum:

$$
T=\frac{I_{t}}{n I_{t, \mathrm{vac}}}
$$

The factor $n$ is the refractive index used at the bottom boundary in the wire simulation.

\section{SIMULATION RESULTS}

Due to the random nature of geometries, many different geometrical arrangements of wires must be simulated, and the extracted results must be averaged to arrive at global conclusions. Also, the wavelengths of the sunlight must be varied. At the time of the conference, an insufficient number of geometrical arrangements have been simulated as to draw global conclusions. We therefore discuss only a few typical results.

We first draw our attention to the photonic properties. Figure 5 shows the photo-generation rate in a horizontal 

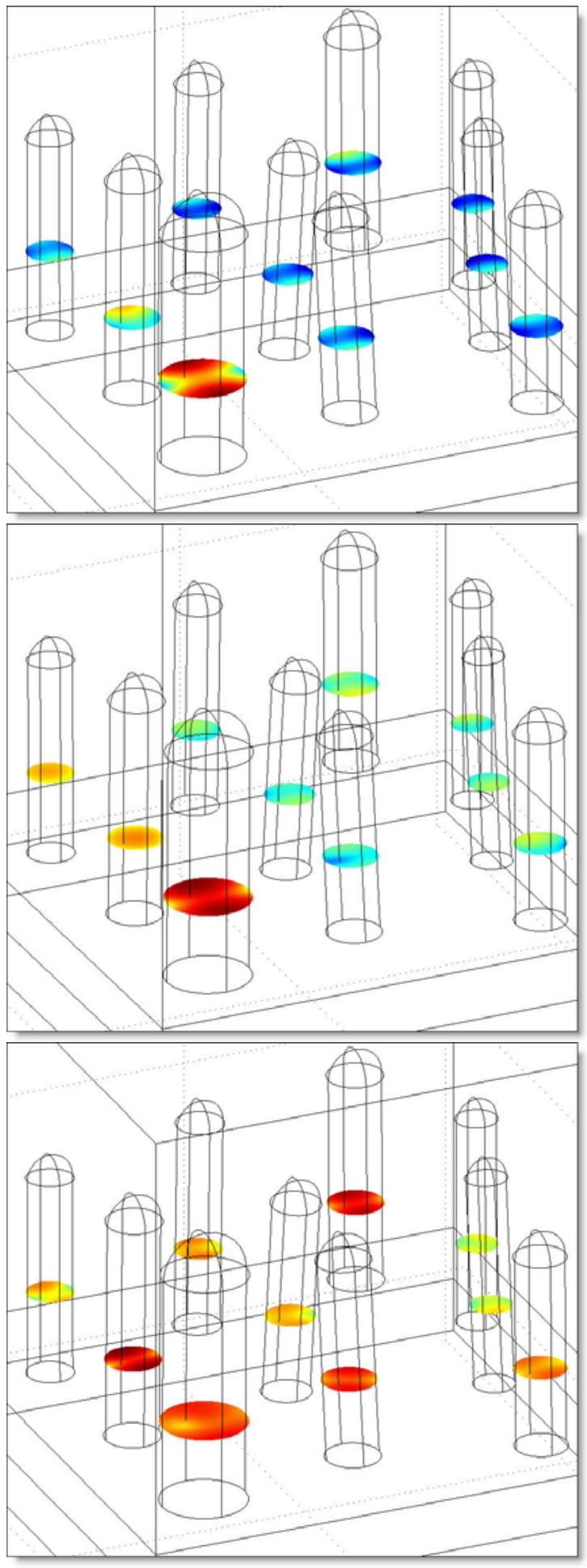

Figure 5. The calculated photo-generation rates of sunlight with normal incidence, having a wavelengths of $600 \mathrm{~nm}$ (top), 800 and $1000 \mathrm{~nm}$ (middle and bottom). Note the various excitation modes. Red $=$ highest rate, blue $=$ lowest rate (The plot range is adjusted at every wavelength).
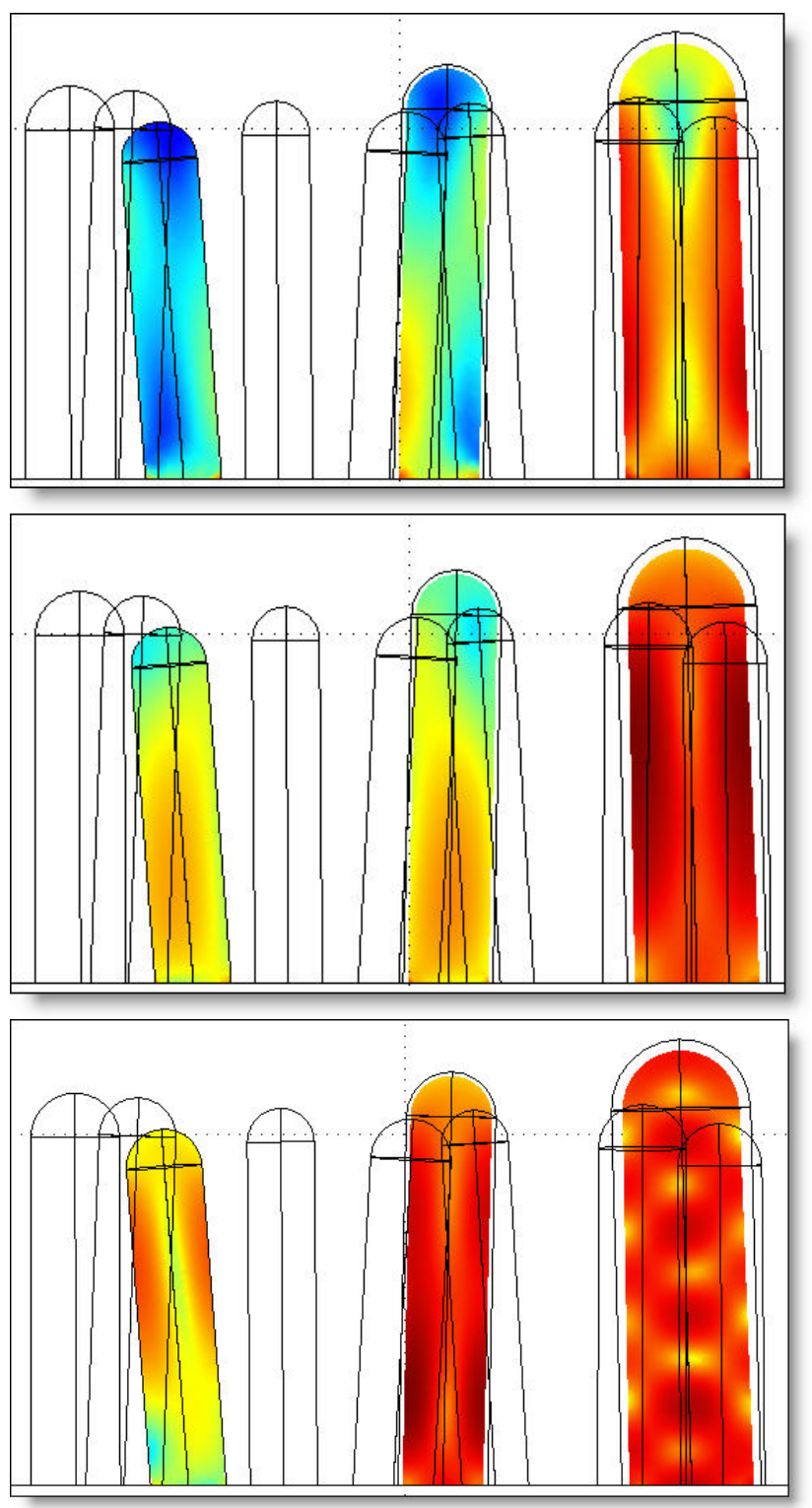

Figure 6. Vertical, cross-sectional view of Figure 5 through the three left-most wires. Shown is the photogeneration rate at wavelengths of $600 \mathrm{~nm}$ (top), 800 and $1000 \mathrm{~nm}$ (middle and bottom). The photo-generation rate is color-indexed in the same way as in Figure 5.

cross-section for various wavelengths $\lambda$ of sunlight. At $\lambda=$ $600 \mathrm{~nm}$ (top panel), mainly the foremost wire with the largest diameter absorbs light, and the cross-sectional distribution of photo-generation points to a photonic mode. A photonic mode in the diffraction limit is the equivalent to a configuration of strong light trapping in the limit of geometrical ray tracing, i.e. photonic modes usually enhance photo-generation strongly. The modes are also obvious in Figure 6, which shows a vertical cross-sectional view through the left-most three wires. Note that the 
photonic mode in the wire with the largest diameter undergoes a transformation from one vertical node at $\lambda=$ $600 \mathrm{~nm}$ to five nodes at $\lambda=1000 \mathrm{~nm}$. Also note on both Figures that the photo-generation becomes more homogeneously spread over all wires with longer wavelength. We observe a rich variety of photonic modes. For example in Figure 7, there are five tori layered above each other. They are slightly asymmetrical because the surroundings of the wire are asymmetrical, and the wire is slightly tilted to the left. So far, we found that photonic modes are stronger in thick $(\approx 200 \mathrm{~nm})$ than in thin $(\approx 100$ $\mathrm{nm})$ wires.

Because sunlight has a continuous spectrum, and the wires are arranged randomly, the photonic modes are completely smeared out in reflectance and transmission measurements. However, they may contribute significantly to the overall (integrated) absorption, possibly even more significantly than scattering.

The absorbance, reflectance and transmittance of a simulation are shown in Figure 8 . The glass substrate is only sparsely covered by nearly upright, thin wires (10 wires per $1 \times 1 \mu \mathrm{m}^{2}$ with a diameter of $\approx 100 \mathrm{~nm}$ ), which causes the absorption to be lower at short wavelengths because more light is reflected at the glass substrate. This is also observed in the quantum efficiency measurements published in [4].

In the following, we compare the absorbance in this wire array with the Lambertian limit of homogeneous, diffuse scattering in a bulk Si film with thickness $W$ and a perfect back mirror [10]:

$$
A=1-\exp \left\lfloor-\alpha 4 n^{2} W\right\rfloor
$$

This limit assumes that all of the isotropically incident rays have a path length that equals the theoretical maximum of $4 n^{2} W$, an optimum that has not been realized. This limit is represented by the dashed line in Figure 8 . Our simulation surpasses this limit at $\lambda=1000 \mathrm{~nm}$ because our excitation light is not incident isotropically. Note, that many experiments (for example [5]) yield $A=1-T-R$ similar to our

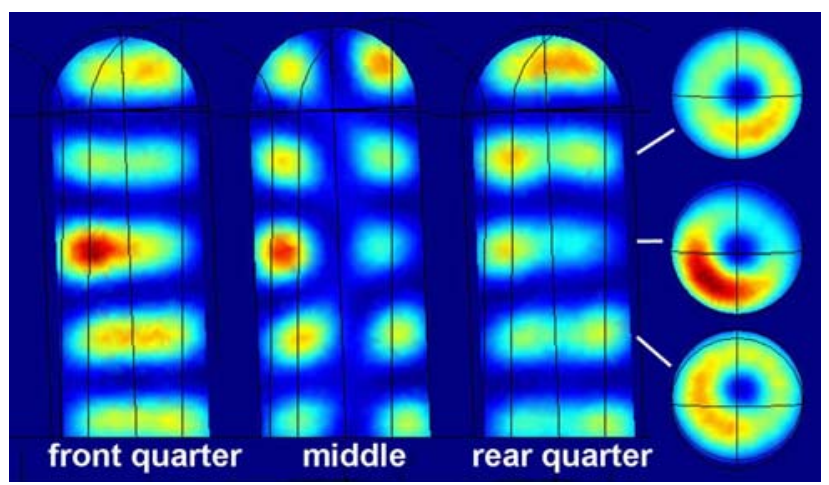

Figure 7. The photo-generation rate in a $200 \mathrm{~nm}$ thick Si wire in sunlight with a wavelength of $600 \mathrm{~nm}$ shows five tori. Left: three different vertical cross-sections along the wire; right: horizontal cross-sections through three tori.

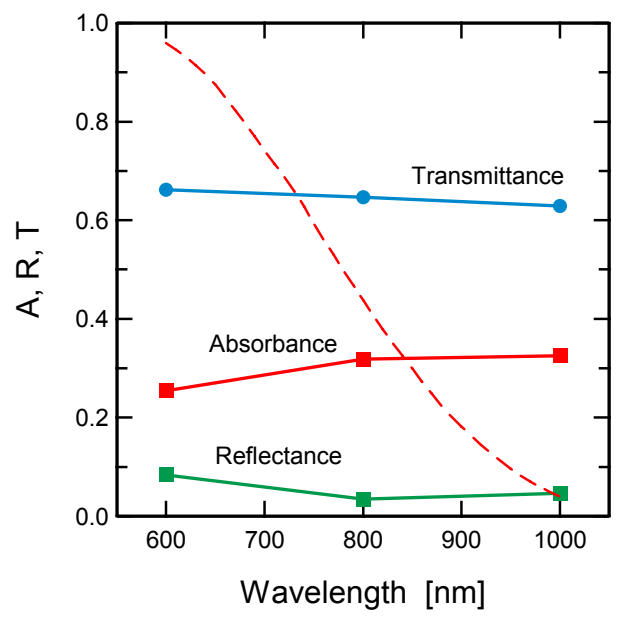

Figure 8. Symbols: absorbance, transmittance, and reflectance extracted from a simulation of ten $\approx 500 \mathrm{~nm}$ long wires with $\approx 100 \mathrm{~nm}$ diameter, positioned in an area of $1 \times 1 \mu \mathrm{m}^{2}$. The sunlight with $\lambda=600 \mathrm{~nm}$ is incident from a right angle above. Dashed line: Lambertian limit of a bulk Si film with $500 \mathrm{~nm}$ thickness.

simulations: the absorbance exceeds the Lambertian limit by a factor of 10 or more at $\lambda=1000 \mathrm{~nm}$. Plasmonic coupling of light has been stated as the reason for this.

\section{CONCLUSIONS}

A model for the solution of Maxwell equations in a volume of $1 \times 1 \times 1.4 \quad \mu^{3}$ with 10 wires has been established, using finite element modeling with the time-harmonic approach. The total number of mesh points in our domain is kept below 400000 , and results in 2.5 million degrees of freedom for solving the Maxwell equations. Hence, a computer system with 128 GB of RAM is sufficient for this task. Due to the random nature of geometries, many different geometrical arrangements of wires must be simulated, and the extracted results must be averaged to arrive at global conclusions. We observe a rich variety of photonic modes, especially within rather tick wires $(\approx 200 \mathrm{~nm})$. For example, our simulations surpass the Lambertian limit (for isotropically incident light) at $\lambda=$ $1000 \mathrm{~nm}$, as has been reported in many experiments.

\section{REFERENCES}

[1] B. M. Kayes, M. A. Filler, M. D. Henry, J. R. Maiolo III, M. D. Kelzenberg, M. C. Putnam, J. M. Spurgeon, K. E. Plass, A. Scherer, N. S. Lewis, H. A. Atwater, "Radial pn junction, wire array solar cell”, 33rd IEEE PVSC, 2008.

[2] O. L. Muskens, J. G. Rivas, R. E. Algra, E. P. A. M. Bakkers, A. Lagendijik, "Design of light scattering in nanowire materials for photovoltaic applications", Nano Letters 8, 2008, pp. 2638 - 2642. 
[3] R. A. Street, P. Qi, R. Lujan, W. S. Wong, "Reflectivity of disordered silicon nanowires", Appl. Phys. Lett. 93, 2008, No. 163109.

[4] L. Tsakalakos, J. Balch, J. Fronheiser, B. A. Korevaar, O. Sulima, J. Rand, "Silicon nanowire solar cells", Appl. Phys. Lett. 91, 2007, No. 233117.

[5] T. Stelzner, M. Pietsch, G. Andrä, F. Falk, E. Ose, S. Christiansen, "Silicon nanowire-based solar cells", Nanotechnology 19, 2008, No. 295203.

[6] M. Metropolis, A. W. Rosenbluth, M. N. Rosenbluth, A. H. Teller, E. Teller, "Equation of state calculations by fast computing machines", J. Chem. Phys. 21, 1953, pp. 1087 $-1092$.

[7] Comsol, Version 3.5, Comsol AB, Stockholm, Sweden, http://www.comsol.com

[8] Yee, K.S., "Numerical solution of initial boundary value problems involving Maxwell's equations in isotropic media," IEEE Transactions on Antennas and Propagation 14, 1966, pp. $302-307$.

[9] P. P. Altermatt, J. Wang, R. Reineke-Koch, R. Brendel, "Optical modeling of nanostructured films for selective coatings", SPIE Solar Energy and Applications, San Diego, CA, 2008, paper no. 704607_1.

[10] R. Brendel, Thin-film crystalline silicon solar cells, Whiley- $\mathrm{VCH}$, Weinheim, Germany, 2003, ISBN: 3-527-40376-0 